Replik des SMB zur vorangegangenen Stellungnahme der AGLA zum Fachbericht «Statine zur Primärprävention kardiovaskulärer Erkrankungen»

\title{
Das Ziel: Gefahren der Überbehandlung und Unterbehandlung vermeiden
}

\section{Urs Metzger ${ }^{a}$,}

\section{Hans Bohnenblust ${ }^{b}$}

Im Namen des Fachgremiums

Swiss Medical Board

a Prof. Dr. med. Dr. h.c. Vorsitzender des Expertenrats

b Dipl.-Ing. ETH, M.S. MIT, (Technology Assessment)
Die Arbeitsgruppe Lipide und Atherosklerose (AGLA) der Schweizerischen Gesellschaft für Kardiologie hat im vorangegangenen Beitrag Stellung zum Fachbericht «Statine zur Primärprävention kardiovaskulärer Erkrankungen» genommen und kritisiert die Vorgehensweise des SMB in einigen Punkten als fehlerhaft und irreführend.

Das SMB möchte an dieser Stelle einige Präzisierungen vornehmen, um zu einem besseren Verständnis des Fachberichtes beizutragen.

Der Trägerverein des SMB setzt sich im Jahr 2014 aus Vertretern der Gesundheitsdirektorenkonferenz GDK, der FMH, der SAMW und der Regierung des Fürstentums Liechtenstein zusammen und ist für die Themenwahl (und die Umsetzung der Empfehlungen) verantwortlich. Der Trägerverein versteht sich jedoch nicht als «Sprachrohr» der konstituierenden Organisationen.

Das von Verwaltung, Leistungserbringern und Industrie unabhängige, interdisziplinäre Fachgremium besteht aus dem Expertenrat und dem Team der Geschäftsstelle. Diese erarbeiten die Fachberichte und formulieren Empfehlungen als Entscheidungsgrundlage zuhanden des Trägervereins. Die Literatursuche und -auswertung, die inhaltliche Bearbeitung der einzelnen Kapitel sowie die Formulierung des Berichts erfolgen durch das Team der Geschäftsstelle. Die Berichtsentwürfe werden durch den Expertenrat geprüft und gemeinsam mit dem Team der Geschäftsstelle im Rahmen mehrerer Workshops des Fachgremiums Swiss Medical Board diskutiert. Die Schlussfassung des Berichts mit den Empfehlungen wird durch den Expertenrat verabschiedet.

\section{«Der Fachbericht behandelt aus- schliesslich die Primärprävention.»}

Korrespondenz: Sekretariat Trägerschaft Swiss Medical Board Stampfenbachstrasse 30 CH-8090 Zürich Tel. 0432595211 info[at]medical-board.ch gewiesene Experten im jeweiligen Themengebiet bei.

Diese themenspezifischen Fachspezialisten werden auf Vorschlag der FMH beigezogen. Sie werden im Rahmen eines Gesprächs zu den medizinischen Aspekten der Fragestellung durch zwei Vertreter des Teams der Geschäftsstelle befragt und nehmen zum medizinischen und ökonomischen Teil der Fachberichte (Kapitel 1 bis 4) Stellung, ob hier eine sachlich korrekte Darstellung erfolgte. Dies ist auch beim vorliegenden Fachbericht erfolgt, bei dem auf Vorschlag

\section{«Das Fachgremium zieht ausge- wiesene Experten im jeweiligen Themengebiet bei.»}

der FMH drei Fachspezialisten konsultiert wurden, darunter auch ein Mitglied der AGLA.

Die Fachspezialisten sind jedoch nicht in die ethischen und rechtlichen Abwägungen, in die Formulierung der Empfehlungen und die Erstellung der Schlussfassung des Berichts eingebunden. Die Fachberichte geben damit insofern nicht notwendigerweise die Meinung der Fachspezialisten (im Sinne einer Interpretation der dargestellten Evidenzlage) wieder.

In der Stellungnahme der AGLA wird des Weiteren kritisiert, dass das Swiss Medical Board aufgrund seiner Darstellung einige Risikogruppen von einer Prävention ausschliessen würde. Offenbar wurde im Fachbericht des SMB nicht ausdrücklich genug ausgeführt, was in diesem Kontext unter Primär- und Sekundärprävention zu verstehen ist. Der Fachbericht behandelt ausschliesslich die Primärprävention. Nicht Gegenstand des Berichts ist die Sekundärprävention, also die Behandlung aller Patienten mit einer kardialen Vorerkrankung oder erheblichen Risikofaktoren für eine solche Erkrankung. Somit sind auch Patienten mit Diabetes mellitus, genetischen Dyslipidämien oder manifesten arteriosklerotischen Veränderungen nicht Gegenstand des Berichts. Die entsprechende Darstellung wird im Fachbericht in diesem Sinne angepasst. 
Dass im Rahmen der kardiologischen Behandlung (und Prävention) alle Risikofaktoren betrachtet werden sollten, ist unbestritten. Allerdings weisen wir darauf hin, dass der Bericht lediglich die Wirksamkeit der Statine in der Behandlung von Dyslipidämien (und damit der Vermeidung künftiger kardialer Ereignisse) betrachtet, jedoch nicht das optimale Vorgehen bei der hausärztlichen oder kardiologischen Konsultation im Allgemeinen, wozu die AGLA die Verwendung ihres «AGLA-Rechners» empfiehlt.

Die AGLA geht in ihrer Stellungnahme von einer deutlichen Kostenüberschätzung in der KostenEffektivitäts-Analyse des SMB aus. Das SMB hat die internationale Studienlage zur Kosteneffektivität einer Statintherapie in der Primärprävention ausführlich dargelegt (S. 23 und 24 des Fachberichtes). berücksichtigen, dass bei den meisten Wirkstoffen (Fluvastatin, Pitavastatin, Pravastatin, Rosuvastatin) mit steigender Dosierung auch der Preis ansteigt. Durch die Praxis der Tablettenteilung wäre zwar eine Kostenreduzierung der Statintherapie zu erreichen (gleiche Compliance der Patienten wie bei Therapie ohne Tablettenteilung vorausgesetzt). Es ist jedoch anzunehmen, dass das Kosten-Wirksamkeits-Verhältnis weiterhin in einen ungünstigen Bereich fallen würde.

Zur Bestimmung der Risikoschwelle, ab der eine Primärprävention mit Statinen sinnvoll ist, verweisen wir in diesem Zusammenhang noch einmal auf die Ausführungen im Fachbericht: «... dass in der Meta-Analyse der Cholesterol Treatment Trialists' Collaborators keiner der beiden Scores [PROCAM, ESC] verwendet wurde. Die Autoren der Meta-Ana-

\section{«Die entsprechende Darstellung wird im Fachbericht in diesem Sinne angepasst.»}

Die Stellungnahme der AGLA hebt hervor, dass das SMB im Bericht eine Effektunterschätzung der Statintherapie vorgenommen habe: Mit der Standardtherapie sei nicht nur eine Senkung des LDLCholesterins um $1 \mathrm{mmol} / \mathrm{l}$, sondern um $2 \mathrm{mmol} / \mathrm{l} \mathrm{zu}$ erreichen, was eine erhebliche Auswirkung auf das Kosten-Wirksamkeits-Verhältnis habe. Das Swiss Medical Board hat seine Effektschätzung aus der CTTCStudie übernommen, die letztlich Grundlage der Berechnungen ist: «22 trials compared a standard statin regimen versus control (134537 participants; mean baseline LDL cholesterol 3.70 [SD 0.7] mmol/l; mean difference at 1 year $1.08 \mathrm{mmol} / \mathrm{l} ;$...». Bei höheren Ausgangswerten ist mit einer Statintherapie (abhängig von der Dosis) sicherlich auch eine höhere Reduktion zu erreichen. Festzuhalten ist jedoch, dass das Kosten-Wirksamkeits-Verhältnis selbst unter der von der AGLA postulierten Effektgrösse immer noch in einen ungünstigen Bereich fallen würde.

Zur von der AGLA postulierten «häufigen Praxis der Teilung von Tabletten» ist uns keine Publikation bekannt, die diese Praxis in der Schweiz quantifizieren würde. In diesem Zusammenhang ist ferner zu lyse haben ein eigenes Berechnungsprinzip für die Bestimmung des kardiovaskulären Risikos entwickelt. Sie ermitteln das 5-Jahres-Risiko für ein kardiovaskuläres Ereignis insgesamt (fatale und nichtfatale Ereignisse zusammen), während der ESC-Score ein 10-Jahres-Risiko für ein tödliches Ereignis ermittelt. Auch wird im ESC-Score das Gesamtcholesterin verwendet, in der Meta-Analyse hingegen das LDLCholesterin. Inwiefern das Berechnungsprinzip in der Meta-Analyse zu einer vergleichbaren Klassifizierung wie der ESC-Score (oder des PROCAM Score) führt, ist noch nicht untersucht oder validiert worden». Diese Problematik konnte auch von der AGLA in ihrer Stellungnahme nicht aufgelöst werden.

Insgesamt sind wir mit der AGLA einig, dass es gilt, sowohl die Gefahren einer Überbehandlung (AHA/ACC-Empfehlungen) und Unterbehandlungen zu vermeiden. Es ist erklärtes Ziel der Empfehlungen des SMB, zu dieser Balance beizutragen. In diesem Sinn hat das SMB diesen Punkt nochmals diskutiert und den Schwellenwert für ein tödliches kardiovaskuläres Ereignis bezogen auf einen Zeithorizont von 10 Jahren auf 7,5\% festgelegt. 